\title{
ÁLAMO CRIOLLO (Populus nigra cv Italica) IMPREGNADO COMO ALTERNATIVA AL USO DE MADERA DE Nothofagus spp EN LA PROVINCIA DE SANTA CRUZ, ARGENTINA
}

\author{
Spavento, Eleana ${ }^{1}$; Keil, Gabriel ${ }^{1}$; Nosetti, Julia²; Monelos, Lucas ${ }^{2}$ y Peri, Pablo 3
}

RESUMEN

En la provincia de Santa Cruz, Argentina, actualmente se utilizan maderas nativas valiosas del género Nothofagus en productos de carpintería rural de bajo valor agregado. Asimismo, en dicha provincia existe un recurso importante de álamo criollo, Populus nigra cv Italica, implantado en cortinas protectoras de cultivos fruti hortícolas y montes de abrigo para ganado. Si bien la madera de dicha especie es utilizada en productos de carpintería rural, su empleo se encuentra limitado a su baja durabilidad natural.

Los ensayos de degradación a campo (cementerios de estacas), permiten evaluar la durabilidad natural o adquirida de una madera expuesta a condiciones de riesgo (edafoclimáticas y biológicas) similares a las que está expuesta una madera en servicio. Estos ensayos son evaluados a través de una categorización del estado de la madera. No obstante ello, resulta interesante realizar una evaluación cuantitativa de la pérdida de resistencia mecánica del material expuesto a la intemperie.

Entre los productos preservantes disponibles en el mercado, la creosota es adecuada para determinados empleos de la madera, por su accesibilidad y su factibilidad de aplicación por baño caliente frío.

El objetivo de este trabajo fue evaluar el comportamiento de la madera de álamo criollo impregnada con creosota, mediante baño caliente frío, y compararla con la madera de lenga (Nothofagus pumilio (Poepp. \& Endl.) Krasser) y de ñire (Nothofagus antartica (G.Forst.) Oerst.), ambos sin preservar y de reconocida durabilidad natural, como así también, con madera de álamo criollo sin impregnar como testigo.

El material de ensayo, provino de 3 cementerios de estacas ubicados en las localidades de Perito Moreno, Gobernador Gregores y Río Gallegos, provincia de Santa Cruz, Argentina, y estuvo expuesto a degradación durante 4 años. Se determinaron: contenido de humedad: $\mathrm{CH}$ (IRAM 9532), densidad aparente normal Dn (IRAM 9544) y módulo de rotura (MOR) en flexión estática (IRAM 9545).

El CH del álamo con creosota fue significativamente superior al de las otras especies en

\footnotetext{
1 Facultad de Ciencias Agrarias y Forestales. Universidad Nacional de La plata. Calle 60 y 119. CP: 1900. CC 31-La Plata, Argentina. eleanaspavento@yahoo.com.ar

3 Universidad Nacional de la Patagonia Austral
} 
los tres sitios de exposición, posiblemente porque en estufa a $103 \pm 2{ }^{\circ} \mathrm{C}$, se hayan evaporado algunos componentes de la creosota. Los valores de Dn y MOR en flexión estática, no presentaron diferencias significativas entre los sitios de exposición, pero sí entre especies y entre álamo criollo con y sin tratamiento preservante.

La madera de álamo criollo impregnada con creosota podría tener prestaciones similares a las de lenga y ñire para usos en productos de carpintería rural.

Palabras clave: Patagonia, creosota, baño caliente frío, cementerio de estacas, flexión estática

\section{SUMMARY}

In the Santa Cruz province, Argentina, native woods of Nothofagus genus species are used in low value rural woodwork products. Also, in this province, there is an important resource of Populus nigra cv Italica as protective curtains to vegetable and fruit crops and as shelter forests for cattle. The wood of this species is also used in rural woodwork products, but the use of this wood is limited because of its low natural durability value.

Natural degradation field trials allow assessing the natural or acquired durability of wood exposed to similar soil, climatic and biological conditions to a wood in service. These tests are evaluated through a wood state categorization. Nevertheless, it is interesting to make a quantitative assessment of the loss of mechanical strength of the material exposed to the weathering.

Among the commercially available preservatives products, creosote is appropriate for certain uses of wood, due to its accessibility and its feasible application by hot-cold bath.

The objective of this study was to evaluate the behavior of Populus nigra cv Italica wood impregnated with creosote, by hot-cold bath, and to compare it to Lenga (Nothofagus pumilio (Poepp. \& Endl.) Krasser) and Ñire (Nothofagus antartica ( G. Forst.) Oerst.) wood, both without treatment but having a recognized natural durability. Also is compared to not treated Populus nigra cv Italica wood, as control.

The test material consists of samples exposed to natural degradation in four years field trials located in Perito Moreno, Gobernador Gregores and Rio Gallegos, province of Santa Cruz, and for these samples moisture content CH (IRAM 9532), normal apparent density Dn (IRAM 9544) and rupture modulus (MOR) in static flexion (IRAM 9545) were determined.

The moisture content of Populus nigra cv Italica with creosote was significantly higher than those of the other species at all three sites of exposure, possibly because at $103 \pm 2{ }^{\circ} \mathrm{C}$ in oven some components of creosote were evaporated. The values of normal apparent density and rupture modulus in static flexion showed no significant differences between the exposure sites, but between species and between Populus nigra cv Italica with and without preservative treatment, differences were registered. 
The creosote treated Populus nigra cv Italica wood may have a similar suitability to the Nothofagus species wood to be used in rural woodwork products.

Keywords: Patagonia, creosote, hot and cold bath, stakes cemeteries, static bending

\section{INTRODUCCION}

En la provincia de Santa Cruz, Argentina, actualmente se utilizan maderas nativas valiosas del género Nothofagus en productos de carpintería rural de bajo valor agregado, entre las que se destacan lenga (Nothofagus pumilio (Poepp. \& Endl.) Krasser) y ñire (Nothofagus antarctica (G. Foster) Oerst.) (Rechene y Gonda, 1992).

La lenga es una especie de importancia en los bosques australes de Argentina y Chile, tanto por su amplia distribución geográfica como por la calidad y valor económico de su madera (Rechene y Gonda, 1992).

El ñire es una especie de amplia distribución en los bosques Andino-patagónicos (SAyDS, 2005), que se caracteriza por su gran plasticidad y cuya importancia dentro de sistemas silvopastoriles radica principalmente en la capacidad productiva ganadera (ovina y bovina) y la posibilidad de obtención de productos madereros (postes, varas y leña) provenientes de las intervenciones silvícolas (Peri et al, 2009).

Asimismo, en dicha provincia existe un recurso importante de salicáceas cultivadas, entre las que se destacan el álamo criollo, Populus nigra cv Italica, el cual se encuentra principalmente como cortinas protectoras de cultivos fruti hortícolas y montes de abrigo para ganado (Amico et al., 2005). Si bien la madera de dicha especie es utilizada en productos de carpintería rural (postes cortos y leña), su empleo se encuentra limitado a su baja durabilidad natural, presentando una vida útil en servicio, inferior a 5 años (Chiani, 1964; 1965; 1967; Tinto y Chiani, 1967; Arreghini, 1978; citados por Díaz et al., 2002; IRAM 9600, 1998).

El proceso de preservación con productos de acción fungicida e insecticida permite prolongar la vida útil de la madera en servicio (JUNAC, 1988). Asimismo, el nivel de retención del preservante necesario para asegurar una vida útil prolongada de la madera, se determina de acuerdo con las condiciones a las que se expone la madera al ataque de agentes destructores (IRAM 9600, 1998).

De acuerdo con esto, sería interesante evaluar la durabilidad de la madera de álamo criollo tratada con algún producto preservante disponible en el mercado, expuesta a diferentes condiciones, como así también evaluar su comportamiento físico - mecánico, a fin de plantear el reemplazo, en usos de carpintería rural de bajo valor agregado, de las maderas valiosas del bosque nativo.

Los ensayos de degradación a campo (cementerios de estacas), permiten evaluar dicha durabilidad, a través de una categorización del estado de la madera, bajo condiciones de riesgo (edafoclimáticas y biológicas) similares a las que esta expuesta una madera en servicio (JUNAC, 1988). No obstante ello, resulta interesante realizar una evaluación cuantitativa de la 
pérdida de resistencia mecánica del material tratado expuesto a la intemperie.

Entre los productos preservantes disponibles en el mercado, la creosota, si bien presenta algunas restricciones de uso (IRAM 9600, 1998), es adecuada para determinados empleos de la madera, por su accesibilidad y su factibilidad de aplicación por baño caliente frío (JUNAC, 1988).

Como hipótesis de trabajo se consideró que la madera de álamo criollo impregnada con creosota podría tener prestaciones similares que aquellas maderas de especies nativas de reconocida durabilidad natural, para uso en productos de carpintería rural.

\section{OBJETIVO}

Evaluar el comportamiento de la madera de álamo criollo impregnada con creosota, mediante baño caliente frío, y compararla con la madera de lenga y de ñire ambos sin preservar y de reconocida durabilidad natural, como así también, con madera de álamo criollo sin impregnar como testigo.

\section{MATERIAL Y MÉTODO}

\section{Material Leñoso}

Se trabajó con muestras de madera de álamo criollo sin impregnar (testigo), álamo criollo impregnada (creosota baño caliente-frío), lenga y ñire, sin impregnar, provenientes de un ensayo de degradación a campo, expuesta durante 4 años, a tres sitios de la provincia de Santa Cruz: Perito Moreno, Gobernador Gregores y Río Gallegos.

En el Cuadro $N^{\circ} 1$ se presentan datos climáticos y particularidades de interés para la caracterización de las áreas de ensayo. 


\section{Cuadro $\mathrm{N}^{\circ} 1$ \\ FICHA DESCRIPTIVA DE LAS ÁREAS EN ESTUDIO}

\begin{tabular}{|c|c|c|c|}
\hline SITIOS & PERITO MORENO & $\begin{array}{l}\text { GOBERNADOR } \\
\text { GREGORES }\end{array}$ & RIO GALLEGOS \\
\hline Coordenadas & $\begin{array}{l}46^{\circ} 51^{\prime}-47^{\circ} 13^{\prime} \text { LS } \\
71^{\circ} 11^{\prime}-70^{\circ} 19^{\prime} \text { LO }\end{array}$ & $\begin{array}{l}48^{\circ} 40^{\prime}-49^{\circ} 12^{\prime} . \text { LS } \\
69^{\circ} 08^{\prime}-70^{\circ} 20^{\prime} \text { LO }\end{array}$ & $\begin{array}{l}51^{\circ} 22^{\prime}-52^{\circ} 00^{\prime} \text { LS } \\
72^{\circ} 26^{\prime}-69^{\circ} 12^{\prime} \text { LO }\end{array}$ \\
\hline Región Natural & Patagonia Extra-andina & Patagonia. Extra-andina & Patagonia. Extra-andina Austral \\
\hline Ordenes de Suelos & En su mayoría entisoles & $\begin{array}{l}\text { En su mayoría } \\
\text { aridisoles }\end{array}$ & $\begin{array}{l}\text { En su mayoría aridisoles y } \\
\text { molisoles. }\end{array}$ \\
\hline Tipo Climático & Templado frío árido & Templado frío árido & $\begin{array}{l}\text { Templado frío, subhúmedo en el } \\
\text { oeste a semiárido en el este. }\end{array}$ \\
\hline $\begin{array}{l}\text { Precipitación Media } \\
\text { Anual }(\mathrm{mm})\end{array}$ & 72,9 & 191,7 & $\begin{array}{c}260 \\
\text { (estación más lluviosa en otoño) }\end{array}$ \\
\hline $\begin{array}{l}\text { Temperatura Media } \\
\text { Anual }\left({ }^{\circ} \mathrm{C}\right)\end{array}$ & 7,8 & 8,5 & 6,9 \\
\hline $\begin{array}{l}\text { Velocidad Media del } \\
\text { Viento }(\mathrm{Km} / \mathrm{h})\end{array}$ & 35 & 45 & 20 \\
\hline
\end{tabular}

(Fuente: Secretaria de Minería de la Nación y Servicio Meteorológico Nacional)

\section{Método de Impregnación}

El método de impregnación baño caliente frío consistió en sumergir la madera en creosota caliente y luego dejar enfriar la madera sumergida. La variación de temperatura entre los dos baños permitió conseguir retenciones y penetraciones adecuadas a la categoría de riesgo R4. (JUNAC, 1988).

\section{Ensayos Mecánicos}

Para la determinación del comportamiento mecánico de las maderas en estudio se realizó el ensayo de flexión estática, para lo cual se trabajó con probetas de $20 \mathrm{~mm} \times 20 \mathrm{~mm}$ x 300 mm (IRAM 9545, 1985), previamente enterradas hasta el 50\% de su longitud durante 4 años.

Se trabajó con un total de 360 probetas, 120 por sitio de degradación (30 por especie), las cuales fueron estacionadas hasta contenido de humedad de equilibrio higroscópico y acondicionadas para la realización del ensayo.

Para este ensayo se utilizó una prensa universal de $5000 \mathrm{Kg}$ (Alfred J. Amsler y Co, Schaffouse - Suisse 7928). Se trabajó en el Laboratorio de Ensayos de Materiales, Facultad de Ingeniería, Universidad Nacional de La Plata (UNLP). 
Con los datos obtenidos se calculó el módulo de rotura (MOR)

\section{Ensayos Físicos}

Para la determinación del comportamiento físico de las maderas, se determinó el contenido de humedad (IRAM 9532, 1963) y la densidad aparente normal (IRAM 9544, 1985), para lo cual, luego de realizado el ensayo de flexión estática, dichas probetas fueron procesadas en carpintería para la obtención de 10 probetas cúbicas de $20 \mathrm{~mm}$ de lado por cada probeta ensayada a flexión estática, con las cuales se realizaron las determinaciones correspondientes.

Se utilizó balanza analítica con una precisión de 0,01g, estufa de secado con termómetro y termostato regulable a $103^{\circ} \mathrm{C} \pm 2^{\circ} \mathrm{C}$, desecador con cloruro de calcio y vaso de precipitado.

Dichos ensayos fueron realizados en los laboratorios del INFIVE y de los cursos de Xilotecnología e Industria de Transformación Mecánica, Departamento de Ingeniería Agrícola y Forestal, Facultad de Ciencias Agrarias y Forestales, UNLP.

Al conjunto de datos de cada variable se le calcularon la media y el coeficiente de variabilidad.

Los datos obtenidos para todas las especies fueron estudiados a partir del Análisis de la Varianza y ante diferencias significativas $(p<0,05)$ se aplicó el test de comparación de medias.

\section{RESULTADOS Y DISCUSIÓN}

En el Cuadro $\mathrm{N}^{\circ} 2$ se presentan los valores de $\mathrm{CH}$, Dn y la comparación de los mismos entre las especies en cada una de las tres localidades en estudio. 


\section{Cuadro $\mathrm{N}^{\circ} 2$ \\ CONTENIDO DE HUMEDAD Y DENSIDAD APARENTE NORMAL EN LAS TRES LOCALIDADES}

\begin{tabular}{|c|c|c|c|c|c|c|}
\hline \multirow{2}{*}{ Especie } & \multicolumn{2}{|c|}{ Gobernador Gregores } & \multicolumn{2}{|c|}{ Perito Moreno } & \multicolumn{2}{|c|}{ Río Gallegos } \\
\hline & $\begin{array}{l}\mathrm{CH} \\
(\%)\end{array}$ & $\begin{array}{c}\text { Dn } \\
(\mathrm{g} / \mathrm{cm} 3)\end{array}$ & $\begin{array}{l}\mathrm{CH} \\
(\%)\end{array}$ & $\begin{array}{c}\text { Dn } \\
(\mathrm{g} / \mathrm{cm} 3)\end{array}$ & $\begin{array}{l}\mathrm{CH} \\
(\%)\end{array}$ & $\begin{array}{c}D n \\
(\mathrm{~g} / \mathrm{cm} 3)\end{array}$ \\
\hline Álamo testigo & $\begin{array}{l}9,22 \mathbf{a} \\
(9,03)\end{array}$ & $\begin{array}{c}0,33 \mathbf{a} \\
(11,17)\end{array}$ & $\begin{array}{c}9,49 \mathbf{a} \\
(13,60)\end{array}$ & $\begin{array}{c}0,37 \mathbf{a} \\
(10,62)\end{array}$ & $\begin{array}{c}9,63 \text { ab } \\
(1,54)\end{array}$ & $\begin{array}{c}0,33 \mathbf{a} \\
(11,16)\end{array}$ \\
\hline Álamo con creosota & $\begin{array}{c}30,11 \mathbf{b} \\
(29,09)\end{array}$ & $\begin{array}{c}0,59 \mathbf{b} \\
(16,96)\end{array}$ & $\begin{array}{c}29,24 \mathbf{b} \\
(11,41)\end{array}$ & $\begin{array}{c}0,63 \mathbf{b} \\
(12,74)\end{array}$ & $\begin{array}{c}24,43 \mathbf{c} \\
(11,58)\end{array}$ & $\begin{array}{c}0,53 \mathbf{b} \\
(12,91)\end{array}$ \\
\hline Lenga & $\begin{array}{c}9,19 \mathbf{a} \\
(13,42)\end{array}$ & $\begin{array}{c}0,55 \mathbf{b} \\
(3,88)\end{array}$ & $\begin{array}{c}10,20 \mathbf{a} \\
(10,25)\end{array}$ & $\begin{array}{l}0,53 \mathbf{c} \\
(6,36)\end{array}$ & $\begin{array}{c}10,04 \mathbf{b} \\
(2,14)\end{array}$ & $\begin{array}{l}0,53 \mathbf{b} \\
(7,85)\end{array}$ \\
\hline Ñire & $\begin{array}{l}9,75 \mathbf{a} \\
(9,01)\end{array}$ & $\begin{array}{c}0,59 \mathbf{b} \\
(11,90)\end{array}$ & $\begin{array}{c}10,22 \mathbf{a} \\
(9,51)\end{array}$ & $\begin{array}{l}0,61 \mathbf{b} \\
(3,14)\end{array}$ & $\begin{array}{c}9,06 \text { ab } \\
(9,14)\end{array}$ & $\begin{array}{c}0,61 \mathbf{b} \\
(15,75)\end{array}$ \\
\hline
\end{tabular}

Diferentes letras denotan diferencias significativas con el test de Tukey $(p<0.05)$.

Los coeficientes de variación (CV\%) se indican entre paréntesis.

El contenido de humedad $(\mathrm{CH})$ se define como la cantidad de agua que tiene la madera con respecto a su masa anhidra (Andía y Keil, 2004). El mismo influye en la determinación de las propiedades físicas y mecánicas ya que modifica el volumen y la masa del material (Coronel, 1994).

Según se observa en el Cuadro $\mathrm{N}^{\circ} 2$, el $\mathrm{CH}$ del álamo con creosota fue significativamente superior con respecto a las otras especies en los tres sitios de exposición, resultando en todos los casos un valor extremadamente alto, presumiblemente por la interferencia que generó la creosota mediante el método de pesadas; es posible que cuando se expuso el material a $103 \pm$ $2{ }^{\circ} \mathrm{C}$, además del agua tecnológica, se hayan evaporado algunos componentes de la creosota con puntos de ebullición inferiores a los valores de temperatura señalados.

La densidad de la madera es la relación entre su peso y su volumen (Coronel, 1994). La densidad aparente (Da) relaciona el peso y el volumen de la madera considerando los espacios porosos: macro y microporos (Coronel, 1994).

La densidad aparente normal (Dn) es la relación existente entre el peso y el volumen de la madera a un determinado contenido de humedad. Dicho valor es importante debido a que es el más estable de la madera en uso y, en consecuencia, es el que se considera para las determinaciones de resistencia mecánica. Su valor se tiene en cuenta en la industria de la madera aserrada y en productos de madera sólida (Andía y Keil, 2004).

La Dn del álamo con creosota no presentó diferencias significativas con respecto a la lenga y el ñire, en los sitios de Gobernador Gregores y Río Gallegos, mientras que en el sitio de Perito Moreno, se diferenció de la lenga pero no del ñire. 
En todos los casos, la creosota elevó la densidad aparente normal en la madera de álamo diferenciándose significativamente de la Dn del álamo testigo.

En todos los sitios, el álamo testigo proporcionó valores de densidad bajos mientras que el álamo con creosota, presentó valores medios al igual que la lenga y el ñire (Rivero Moreno, 2004).

\section{Cuadro $\mathrm{N}^{\circ} 3$}

\section{VALORES DE CH Y DE DN COMPARADO ENTRE SITIOS}

\begin{tabular}{|c|c|c|c|c|c|c|c|c|}
\hline \multirow{2}{*}{ Sitio } & \multicolumn{2}{|c|}{ Álamo Testigo } & \multicolumn{2}{|c|}{$\begin{array}{l}\text { Álamo con } \\
\text { Creosota }\end{array}$} & \multicolumn{2}{|c|}{ Lenga } & \multicolumn{2}{|c|}{ Ñire } \\
\hline & $\begin{array}{l}\mathrm{CH} \\
(\%)\end{array}$ & $\begin{array}{c}D n \\
\mathrm{Kg} / \mathrm{m} 3)\end{array}$ & $\begin{array}{l}\mathrm{CH} \\
(\%)\end{array}$ & $\begin{array}{c}\text { Dn } \\
(\mathrm{Kg} / \mathrm{m} 3)\end{array}$ & $\begin{array}{l}\mathrm{CH} \\
(\%)\end{array}$ & $\begin{array}{c}\text { Dn }(\mathrm{Kg} / \\
\mathrm{m} 3)\end{array}$ & $\begin{array}{l}\mathrm{CH} \\
(\%)\end{array}$ & $\begin{array}{c}\text { Dn } \\
(\mathrm{Kg} / \mathrm{m} 3)\end{array}$ \\
\hline $\begin{array}{l}\text { Gobernador } \\
\text { Gregores }\end{array}$ & $\begin{array}{l}9,22 \mathbf{a} \\
(9,03)\end{array}$ & $\begin{array}{c}330 \mathbf{a} \\
(11,17)\end{array}$ & $\begin{array}{l}30,11 \mathbf{a} \\
(29,09)\end{array}$ & $\begin{array}{c}590 \mathbf{a} \\
(16,96)\end{array}$ & $\begin{array}{c}9,19 \mathbf{a} \\
(13,42)\end{array}$ & $\begin{array}{l}550 \mathbf{a} \\
(3,88)\end{array}$ & $\begin{array}{l}9,75 a \\
(9,01)\end{array}$ & $\begin{array}{c}590 \mathbf{a} \\
(11,90)\end{array}$ \\
\hline Perito Moreno & $\begin{array}{c}9,49 \mathbf{a} \\
(13,60)\end{array}$ & $\begin{array}{c}370 \mathbf{a} \\
(10,62)\end{array}$ & $\begin{array}{l}29,24 \mathbf{a} \\
(11,41)\end{array}$ & $\begin{array}{c}630 \mathbf{a} \\
(12,74)\end{array}$ & $\begin{array}{r}10,20 \mathbf{a} \\
(10,25)\end{array}$ & $\begin{array}{l}530 \mathbf{a} \\
(6,36)\end{array}$ & $\begin{array}{c}10,22 \mathbf{a} \\
(9,51)\end{array}$ & $\begin{array}{c}610 \mathbf{a} \\
(3,14)\end{array}$ \\
\hline Río Gallegos & $\begin{array}{l}9,63 \mathbf{a} \\
(1,54)\end{array}$ & $\begin{array}{c}330 \mathbf{a} \\
(11,16)\end{array}$ & $\begin{array}{l}24,43 \mathbf{b} \\
(11,58)\end{array}$ & $\begin{array}{c}530 \mathbf{a} \\
(12,91)\end{array}$ & $\begin{array}{c}10,04 \mathbf{b} \\
(2,14)\end{array}$ & $\begin{array}{l}530 \mathbf{a} \\
(7,85)\end{array}$ & $\begin{array}{l}9,06 \mathbf{a} \\
(9,14)\end{array}$ & $\begin{array}{c}610 \mathbf{a} \\
(15,75)\end{array}$ \\
\hline
\end{tabular}

Diferentes letras denotan diferencias significativas con el test de Tukey $(p<0.05)$.

Los coeficientes de variación (CV\%) se indican entre paréntesis.

Según se observa en el Cuadro $\mathrm{N}^{\circ} 3$, los valores de $\mathrm{CH}$ para el álamo testigo, la lenga y el ñire no presentaron diferencias significativas entre sitios. El CH del álamo con creosota fue significativamente menor en Rio Gallegos con respecto a los otros dos sitios.

Los valores de Dn para el álamo testigo, el álamo con creosota, la lenga y el ñire no se diferenciaron entre sitios.

En el Cuadro $\mathrm{N}^{\circ} 4$ se presentan los valores de MOR comparando entre las especies en cada una de las tres localidades en estudio. 


\section{Cuadro $\mathrm{N}^{\circ} 4$ \\ RESULTADOS DEL ENSAYO DE FLEXIÓN ESTÁTICA EN GOBERNADOR GREGORES, PERITO MORENO Y RÍO GALLEGOS}

\begin{tabular}{|c|c|c|c|}
\hline \multirow{2}{*}{ Especie } & Gobernador Gregores & Perito Moreno & Río Gallegos \\
\cline { 2 - 4 } & $\begin{array}{c}\text { MOR } \\
(\mathbf{N} / \mathbf{m m} \mathbf{2})\end{array}$ & $\begin{array}{c}\text { MOR } \\
(\mathbf{N} / \mathbf{m m} \mathbf{2})\end{array}$ & $\begin{array}{c}\text { MOR } \\
(\mathbf{N} / \mathbf{m m} \mathbf{2})\end{array}$ \\
\hline \multirow{2}{*}{ Álamo Testigo } & $\begin{array}{c}19,50 \mathbf{a} \\
(20,18)\end{array}$ & $\begin{array}{c}36,30 \mathbf{a} \\
(42,10)\end{array}$ & $\begin{array}{c}27,32 \mathbf{a} \\
(11,71)\end{array}$ \\
\hline \multirow{2}{*}{ Álamo con Creosota } & $44,96 \mathbf{a b}$ & $38,51 \mathbf{a}$ & $44,07 \mathbf{a b}$ \\
$(23,07)$ & $(23,09)$ & $51,81)$ \\
\hline \multirow{2}{*}{ Lenga } & $70,81 \mathbf{b}$ & $64,51 \mathbf{b}$ & $(10,28)$ \\
\hline Nire & $(20,02)$ & $(23,96)$ & $61,70 \mathbf{b}$ \\
& $59,54 \mathbf{b}$ & $71,47 \mathbf{b}$ & $(16,78)$ \\
\hline
\end{tabular}

Letras diferentes denotan diferencias significativas con el test de Tukey $(p<0.05)$.

Los coeficientes de variación (CV \%) se indican entre paréntesis.

EI MOR corresponde a la tensión unitaria máxima en flexión que soporta un material antes de que se produzca la falla, cualquier incremento de carga sobre el material provocará la rotura del mismo (Coronel, 1995).

Del Cuadro № 4 se desprende que en Gobernador Gregores, el álamo impregnado no presentó diferencias significativas entre especies para los valores de MOR. El álamo testigo se diferenció significativamente de la lenga y del ñire pero no se diferenció con el álamo impregnado.

Para el sitio de Perito Moreno, el álamo testigo y el álamo impregnado con creosota, no presentaron diferencias entre sí, pero fueron significativamente menores con respecto a la lenga y el ñire, los cuales no se diferenciaron entre sí para los valores de MOR.

En el caso de Río Gallegos, no existieron diferencias significativas entre el álamo impregnado, la lenga y el ñire, como así tampoco entre el álamo testigo, el álamo con creosota y la lenga.

EI MOR de la madera de álamo criollo testigo y de álamo impregnado fue muy bajo en todas las condiciones a las que estuvo expuesta, mientras que los valores de MOR de la madera de lenga y de ñire, resultaron bajos en todas las condiciones (Rivero Moreno, 2004). 


\section{Cuadro $\mathrm{N}^{\circ} 5$ \\ RESULTADOS DEL ENSAYO DE FLEXIÓN ESTÁTICA COMPARADO ENTRE SITIOS}

\begin{tabular}{|l|c|c|c|c|}
\hline Sitio & Álamo Testigo & Álamo con Creosota & Lenga & Nire \\
\hline & \multicolumn{3}{|c|}{ MOR (N/mm2) } \\
\hline \multirow{2}{*}{ Gobernador Gregores } & $\begin{array}{c}\text { 19,50 a } \\
(20,18)\end{array}$ & $\begin{array}{c}44,96 \mathbf{a} \\
(23,07)\end{array}$ & $\begin{array}{c}70,81 \text { a } \\
(20,02)\end{array}$ & $\begin{array}{c}59,54 \text { a } \\
(59,98)\end{array}$ \\
\hline \multirow{2}{*}{ Perito Moreno } & $36,30 \mathbf{a}$ & $38,51 \mathbf{a}$ & $64,51 \mathbf{a}$ & 71,47 a \\
& $(42,10)$ & $(23,09)$ & $(23,96)$ & $(14,72)$ \\
\hline \multirow{2}{*}{ Río Gallegos } & 27,32 a & $44,07 \mathbf{a}$ & 56,18 a & 61,70 a \\
& $(11,71)$ & $(27,81)$ & $(10,28)$ & $(16,78)$ \\
\hline
\end{tabular}

Letras diferentes denotan diferencias significativas con el test de Tukey $(p<0.05)$.

Los coeficientes de variación (CV \%) se indican entre paréntesis.

Según se indica en el Cuadro $N^{\circ} 5$, no existen diferencias significativas para los valores de MOR entre las tres localidades estudiadas, pese a sus diferencias edáficas y climáticas.

Debido a la variabilidad de algunos datos (altos CV\%), sería aconsejable aumentar el número de repeticiones en posteriores estudios, aunque también se debe tener en cuenta que existen altas variabilidades debido a la multiplicidad de factores intervinientes.

\section{CONCLUSIONES}

En términos generales, los valores de contenido de humedad, densidad aparente normal y módulo de rotura en flexión estática no fueron influenciados por las condiciones de sitio a las que estuvieron expuestos, pero sí por las especies y tratamiento impregnante utilizado.

Según las propiedades tecnológicas, la madera de álamo criollo impregnada con creosota, ofrece prestaciones similares a aquellas maderas de especies nativas de reconocida durabilidad natural, pudiendo ser utilizada en productos de carpintería rural.

De acuerdo con esto, es factible tecnológicamente, el reemplazo de las maderas valiosas del bosque nativo, por madera de álamo criollo impregnado con creosota, pudiendo utilizar dichas especies en usos más adecuados y de mayor valor agregado. 


\section{REFERENCIAS}

Amico, I.; Bava, J. y Calderón, A., 2009. Tabla de volumen para Populus nigra cv Italica en plantaciones lineales en el noroeste de Chubut. 7 pp. Disponible en: ww.sagpya.gov.ar.

Andía, I. y Keil, G., 2004. Propiedades físicas de la madera. Publicación Docente № 01/04. Cátedra de Tecnología de la Madera. Universidad Nacional del Comahue. 28 pp.

Coronel, E. O., 1994. Fundamentos de las propiedades físicas y mecánicas de la madera. Aspectos teóricos y prácticos para la determinación de las propiedades y sus aplicaciones. 1 Parte: Fundamentos de las propiedades físicas de la madera. Publicación ITM - UNSE. 187 pp.

Coronel, E. O., 1995. Fundamentos de las propiedades físicas y mecánicas de las maderas. Aspectos teóricos y prácticos para la determinación de las propiedades y sus aplicaciones. 2 Parte: Fundamentos de las propiedades mecánicas de las maderas. Publicación ITM - UNSE. $335 \mathrm{pp}$.

Díaz, B. G, Luna, M. L. Keil, G. D., Otaño, M.E. y Peri, P. L., 2002. Comportamiento de la madera de Populus nigra cv Italica ante la preservación con creosota y CCA. Revista Investigación Agraria: Sistemas y Recursos Forestales. Vol. 11 (2). 14 pp.

IRAM 9532, 1963. Método de determinación de humedad. Instituto de Racionalización de Materiales. 14 pp.

IRAM 9544, 1985. Método para la determinación de la densidad aparente. Instituto Argentino de Racionalización de Materiales. 10 pp.

IRAM 9545, 1985. Maderas: Método de ensayo de flexión estática. Instituto Argentino de Racionalización de Materiales 10 pp.

IRAM 9600, 1998. Preservación de maderas: Maderas preservadas mediante procesos con presión en autoclave. Instituto Argentino de Racionalización de Materiales. 23 pp.

JUNAC, 1988. Junta del Acuerdo de Cartagena (JUNAC). Manual del Grupo Andino para la Preservación de Maderas. 1프. Ed., Lima. Perú. Pp 294.

Peri, P.; Hansen, N.; Rusch, V.; Tejera, L.; Monelos, L.; Fertig, M.; Bahamonde, H. y Sarasola, M., 2009. Pautas de manejo de sistemas silvopastoriles en bosques nativos de Nothofagus antarctica (ñire) en Patagonia. 1er. Congreso Nacional de Sistemas Silvopastoriles. Ecología. Fisio-ecología. 5 Pp.

Rechene, C. y Gonda, H., 1992. Propuesta metodológica y plan de manejo preliminar para lenga en sitios de calidad media de Chubut. Actas del seminario de manejo forestal de la lenga y aspectos ecológicos relacionados. CIEFAP. Publicación técnica № 8.pp 126-159.

Rivero Moreno, J. 2004. Propiedades Físico-Mecánicas de Gmelina arborea Roxb. y Tectona 
ÁLAMO CRIOLLO (Populus nigra cv Italica) IMPREGNADO COMO ALTERNATIVA AL USO DE MADERA DE Nothofagus spp EN LA PROVINCIA DE SANTA CRUZ, ARGENTINA

grandis Linn. F. Proveniente de Plantaciones Experimentales del Valle del Sacta - Cochabamba. Cochabamba. Bolivia. http://www.monografias.com. 73 pp.

SAyDS, 2005. Primer Inventario Nacional de Bosques Nativos. Ministerio de Salud y Ambiente de la Nación Secretaría de Ambiente y Desarrollo Sustentable, Buenos Aires, pp. 86. 